\title{
Population ageing research: a family of disciplines
}

\author{
Ronald P. Stolk $\cdot$ Inge Hutter $\cdot$ Rafael P. M. Wittek
}

Received: 25 August 2009/Accepted: 10 October 2009/Published online: 28 October 2009

(C) The Author(s) 2009. This article is published with open access at Springerlink.com

\begin{abstract}
To study life course trajectories and ageing, scientific expertise is needed beyond epidemiology. More specifically, appropriate models of life course require a theoretical micro-foundation, need to incorporate multilevel context conditions and the interplay between them. It also requires the application of additional social scientific research methods that go beyond the application of statistical methods based on the multi-stage life table. These research theories and methods are available in disciplines like sociology, cultural anthropology, psychology, demography and economics. To effectively study healthy ageing of populations the individual approach of epidemiology has to be extended with the macro-population and socio-cultural approach of (social) demography and the institutional and network approaches of sociology.
\end{abstract}

Keywords Demography $\cdot$ Epidemiology $\cdot$ Sociology · Ageing

All authors contributed equally.

R. P. Stolk · I. Hutter · R. P. M. Wittek

Healthy Ageing: Population and Society HAPS, University

of Groningen, Groningen, The Netherlands

R. P. Stolk $(\bowtie)$

Department of Epidemiology, University Medical Center Groningen, PO Box 30.001, Groningen, The Netherlands e-mail: R.P.Stolk@epi.umcg.nl

I. Hutter

Department of Demography, University of Groningen, Groningen, The Netherlands

R. P. M. Wittek

Department of Sociology, University of Groningen, Groningen,

The Netherlands
As Bonneux correctly described in his recent commentary "Medical demography and epidemiology: dizygote twins", epidemiology has much in common with medical demography [1], with their shared interest in the study of events in the life course, in particular ageing. However, to study life course trajectories and ageing, more scientific expertise is needed than epidemiology and medical demography. More specifically, we suggest that appropriate models of life course require a better theoretical micro-foundation, need to incorporate multi-level context conditions and the interplay between them. It also requires the application of additional social scientific research methods that go beyond the application of statistical methods based on the multistage life table. Demography is not only the statistical analysis of population, as claimed by Bonneux, but consists of both analytical (macro-population and micro-individual level) and social or cultural demography. The latter combines demographic methods with theories and research methodologies from sociology, cultural anthropology, psychology, and economics.

To effectively study healthy ageing of populations the individual approach of epidemiology has to be extended with the macro-population and socio-cultural approach of (social) demography and the institutional and network approaches of sociology. At the University of Groningen this has recently put into practice in the multi-disciplinary research program for Healthy Ageing, Population and Society (HAPS). The epidemiological population-based cohort study LifeLines [2] will be enriched from these disciplines to better investigate healthy ageing.

Rather than orphan twins, a larger family is needed to resolve the multi-level questions of unhealthy ageing. In what follows, we will first elaborate on two complementary concepts of health and subjective well-being over the life course and how to conceptualize multi-level context 
conditions of ageing. In a second step, we discuss the importance of embedding the life course methodology within society, the required application of both quantitative and qualitative research methods and the need for theoretical micro-foundations, as well as the contribution of epidemiological methods. We conclude with suggestions for a multi-disciplinary research agenda on healthy ageing.

\section{Ageing: health and subjective well-being}

Healthy ageing is a life long endeavor. Chronic multifactorial diseases originate early in life, for many disorders already during pregnancy and adolescence. Symptoms become noticeable at middle age and result in impairments in the elderly [3]. Societal factors affect an individual's life course right from the start. As Bonneux [1] correctly stated, life course can be seen as being started at the moment two future parents meet, the outcome of a complicated social and individual-emotional process. In the prenatal period (smoking, alcohol, maternal diet, medicine use) and postnatal period (circumstances during delivery, breast feeding) societal factors (like number of other children, hours of work of both parents) are important for a successful start of the life course.

Health being defined by the WHO as a state of complete physical, mental and social well-being and not merely the absence of disease or infirmity. The concept of subjective well-being refers to people's own evaluations, both affective and cognitive, of their lives. This relates to the perceived quality of life and consists of self-acceptance, positive relations with others, autonomy, environmental mastery, purpose in life, and personal growth [4]. Subjective well-being is culturally defined, embedded in values of a society, and varies over the life course constituted by stages of development, including the stage of being-elderly [5]. Similarly, stages in the life course are based on culturally interpretations and values that vary between different socio-economic groups and cultural meaning systems in societies. In our approach to healthy ageing we refer to both health and subjective well-being.

\section{Multi-level contexts: cross-level misalignments}

People are connected, so their health is connected. This means that society affects individual's healthy ageing, and individual health influences society. For example, an ageing population has consequences for the labor market, the housing market, pensions to be paid, and health care and support to be provided. At the other hand, elderly people have specific needs and demands regarding their housing, (health) care and support, but also regarding their daily activities (voluntary work; taking care of grandchildren; leisure activities).

Identification of important and modifiable societal determinants is beyond the scope of epidemiology. The same is true for the identification of critical factors at later stages of the life course. Selection of friends, changes during adolescence, coping with life events, adequate treatment of chronic diseases and sufficient networks at old age are examples of potential pitfalls for healthy ageing. An example is self-regulation, that is a person's capacity to plan, guide and monitor her/his own behavior. Elderly persons with higher proactive self-management capacities report higher levels of life satisfaction, positive affect, and well-being [6]. This is strongly affected by context conditions at different levels, such as life events and life style, the embedding in social networks such as family, peers and friends, and in institutions such as health care facilities.Healthy ageing requires physical, mental, and social well-being. These are increasingly affected by the interplay of factors at multiple societal levels:

- at the macro level, national institutions regulate access to and quality of prevention, care, and pensions, while population dynamics (e.g. migration) and demographic processes (e.g. declining fertility rates) affect the opportunities and constraints for different institutional solutions.

- at the meso level, informal communities and social networks as well as formal organizations can provide crucial resources for social and material support, thus constituting essential elements for prevention and mitigation of health related problems. Also meso level data from living and working conditions should be included like distances to family and health care service, but also the amount of green in the living environment.

- at the micro level, individuals do not only differ in their health behavior, physical (e.g. co-morbidity, genetics) and mental predispositions (e.g. self-management ability), but also in disease related risk factors accumulating during their life course. These inter-individual differences, in turn, affect the effectiveness of different preventive measures and interventions.

A key obstacle to successful ageing is misalignment among and across these different levels. Examples for cross-level misalignments are abound: health care routines and structures at meso level are often not equipped to adequately deal with co-morbidity at individual level and changes in age distribution at macro level; macro-level policies building on social support and care for elderly from within their own social network collide with decreasing size and density of personal networks due to labor migration; at meso level, programs for integrated care often fail due to 
inadequate institutional regulations, resulting in interorganizational coordination problems and perverse incentives for care providers. Further research is needed to both find determinants and models of this misalignment, as well as identify factors that predict the misalignment, both at individual and societal level.

Until now, the majority of efforts to investigate the conditions for healthy ageing and devise appropriate interventions neglected this interplay between societal levels and the resulting problems of misalignment. Indeed successful ageing research initiatives have a multidisciplinary approach, like the European Research Area in Ageing (http://era-age.group.shef.ac.uk/) and The Behavioral and Social Research Program at the National Institute on Aging (http://agingcenters.org/).

\section{Healthy ageing: advanced life course methodologies}

There is no doubt that the multi-state life table- "the main demographic tool of the twenty-first century", according to Bonneux [1] - proved to be extremely useful for a more fine-grained representation and modeling of life courses. However, as is the case with all statistical methods, multistage life tables are a methodological device, the use of which depends entirely on the theoretical objectives that they are supposed to serve. Multi-stage life tables can contribute to answer some questions relevant to the subject of healthy aging, but certainly not all of them, and the methodological toolbox relevant for ageing research meanwhile is fortunately much larger than Bonneux' focus on the multi-stage life table suggests, ranging from a variety of anthropological qualitative data collection techniques over multi-level social network methods to structural equation modeling.

Life course research is characterized by longitudinal studies, preferably starting before birth, with repeated measurements over time. To investigate the macro and meso societal levels in healthy ageing, socio-cultural and economic factors on group level have to be added to individual longitudinal assessments. Classical epidemiological studies are transferred in real life course investigations through longitudinal studies of aging which build on life-course theories and event-history models [7]. Ideally studies should cover the entire human life span, but a more feasible approach is including more generations [8]. This introduces both temporal and hierarchical relationships in the data. Standard epidemiological data analyses are useful as first step like multivariate regression techniques, including Cox' proportional hazard analyses, and multi-level models. To fully appreciate the role of different societal levels in healthy ageing, more complex techniques are required like a combination of multi-stage models life table and path analyses. This goes beyond the standard multivariate models and requires the identification of patterns of interpersonal relationships and how these change over the life course. This approach is not limited to psychological or sociological aspects of ageing, as illustrated in a recent paper showing that obesity spreads along social networks [9].

To identify causal determinants and related mechanisms through which they affect healthy aging, potential confounders have to be identified at different levels, ranging from socioeconomic status to number of friends and presence of nearby caregivers. Apart from measures at individual level, confounders can be defined at the meso or macro societal levels. To estimate these complicated associations, multilevel approaches to structural equation models are needed.

To study the cultural meaning attached to subjective well-being in stages in the lifecourse, qualitative research methods are to be applied. Qualitative research methods such as in-depth interviews and focus group discussions provide detailed information on perceptions on health and disease, well-being and happiness, on being elderly. Through qualitative research methods, the voices of people themselves are heard [10]. Surveys such as the World Value Survey provide information on values prevalent at societal level [11].

\section{Micro-foundations: theorizing}

This all needs proper theorizing. By proper theorizing we mean theory that specifies the actor-level micro-foundations and explicates assumptions about how situational opportunities and constraints affect individual level social motives and the resulting individual level outcomes (e.g. health related behavior). In many studies on health and ageing, assumptions about what drives individual decisions are either left implicit or build on a simplified model of human nature, in which individuals are treated as farsighted rational decision makers who make responsible choices about all things that are important to them, including their pensions or health related behavior. Research and policy making in this tradition considered ('irrational') deviations from this model as idiosyncratic and random errors ('noise') at the individual level which would cancel each other out at the population level-yielding an overall 'rational' outcome.

In the light of all the evidence collected during the past two decades by a variety of disciplines ranging from behavioral economics to cognitive neurosciences and cognitive anthropology [12,13], this model of behavior is increasingly difficult to defend. Many of these studies convincingly demonstrate that what has been treated as 
random deviations from rational decision making in fact are systematic and predictable reflections of what has been termed social rationality [14]. The model of social rationality provides a more accurate and realistic micro-foundation of human behavior, in particular with regard to health and well-being. Many ingredients of this new model of human nature are meanwhile widely accepted, like human's stronger reaction to losses than to gains of the same amount, our inclination to give into short term rewards even if we know they are detrimental in the long run, or the strong influence of social comparison and relative status.

Without a proper micro-foundation and the specification of micro-level mechanisms, it will be virtually impossible to assess how macro, meso, and individual level context conditions differentially affect processes and outcomes of aging processes. Behavioral micro-foundations are also essential for identifying and mitigating cross-level misalignments.

In conclusion, not a pair of twins but a family of disciplines, embedded in theoretical micro-foundations, is needed to investigate the multilevel process of healthy ageing. This family will identify relevant factors to explain and predict healthy ageing at macro, meso, and individuallevel.

Open Access This article is distributed under the terms of the Creative Commons Attribution Noncommercial License which permits any noncommercial use, distribution, and reproduction in any medium, provided the original author(s) and source are credited.

\section{References}

1. Bonneux LG. Medical demography and epidemiology: dizygotic twins. Eur J Epidemiol. 2009;24(4):157-9.

2. Stolk RP, Rosmalen JG, Postma DS, de Boer RA, Navis G, Slaets $\mathrm{JP}$, et al. Universal risk factors for multifactorial diseases:
LifeLines: a three-generation population-based study. Eur J Epidemiol. 2008;23(1):67-74.

3. Gluckman PD, Hanson MA, Cooper C, Thornburg KL. Effect of in utero and early-life conditions on adult health and disease. N Engl J Med. 2008;359(1):61-73.

4. Triandis HC. Cultural syndromes and subjective well-being. In: Diener E, Suh EM, editors. Culture and subjective well-being. Cambridge. UK: Bradford Book; 2000. p. 13-36.

5. Suh EM. The self between culture and subjective well-being. In: Diener E, Suh EM, editors. Culture and subjective well-being. Cambridge. UK: Bradford Book; 2000. p. 64-86.

6. Steverink N, Lindenberg S. Do good self-managers have less physical and social resource deficits and more well-being in later life? Eur J Ageing. 2008;5:181-90.

7. Mills M. Individualization and the life course: towards a theoretical model and empirical evidence. In: Howard C, editor. Contested individualization. Toronto: Palgrave MacMillan; 2007. p. 61-79.

8. Ben-Shlomo Y, Kuh D. A life course approach to chronic disease epidemiology: conceptual models, empirical challenges and interdisciplinary perspectives. Int J Epidemiol. 2002;31(2):285-93.

9. Christakis NA, Fowler JH. The spread of obesity in a large social network over 32 years. N Engl J Med. 2007;357(4):370-9.

10. Hennink M, Hutter I, Bailey A. Qualitative research methods. London: Sage; 2009.

11. Inglehart R, Baranez M, Diez-Medrana J, Halman L, Luijkx R. Human beliefs and values. A cross-cultural sourcebook on the 1999-2000 values surveys. Mexico-city: Siglo XXI; 2004.

12. Thaler R. Sunstein C. Wealth, and Happiness. Yale University Press;: Improving Decisions about Health; 2008.

13. Ariely D. Predictably irrational: The hidden forces that shape our decisions. New York: Harper Collins; 2008.

14. Lindenberg S. Social rationality versus rational egoism. In: Turner J, editor. Handbook of sociological theory. New York: Kluwer/Plenum; 2001. p. 635-68. 